\title{
Eccentric Resistance Training and Muscle Hypertrophy
}

\section{Fernanda de Souza-Teixeira ${ }^{1,2 *}$ and Jose Antonio de $\mathrm{Paz}^{2}$}

${ }^{1}$ Superior School of Physical Education, Federal University of Pelotas, Brazil

${ }^{2}$ Institute of Biomedicine, University of León, Spain

\begin{abstract}
Resistance exercise is a key component of any training program aiming to increase strength or muscle mass, regardless of final training goal (e.g. athletic performance, rehabilitation, healthy lifestyle). Great number of studies emphasizes the importance of eccentric muscle actions as a key component of resistance training programs. Thus, resistance eccentric training programs have been compared with other training paradigms analyzing maximum strength manifestations, metabolic cost, muscle activation and fiber recruitment pattern, hypertrophy, as well perceived fatigue. On the other hand, it is well known that the muscle damage and pain induced by eccentric exercise is higher when compared to other training modalities (e.g. concentric exercise). Besides the higher muscle damage, most studies involving eccentric training programs are suggesting greater muscle mass and muscular strength gains with shorter periods of training. The aim of this brief review is to point out the benefits of eccentric training programs, highlighting its effects on muscle hypertrophy, and to indicate the difficulties that can rise when employing such a model.
\end{abstract}

Keywords: Exercise; Cross sectional area; Resistance training; Lengthening

\section{Background}

\section{Eccentric Muscle Actions}

Resistance training programs usually include exercises involving different muscle actions generated through the tension produced by an external load (e.g. eccentric, lengthening; concentric, shortening; and isometric, no change in muscle length). Eccentric (ECC) actions are characterized by development of tension during muscle lengthening [1] and deserve special consideration from the standpoint of physiology, adaptation and training [2].

More than sixty years ago, Katz and Hill reported that force steeply rises when skeletal muscle is lengthened at increasing velocities (ECC contractions), whereas force sharply declines when skeletal muscle is shortened at increasing velocities (concentric contractions). These differences resulted in up to five-fold greater force production in ECC muscle actions if compared with concentric (CON) contractions [3].

\section{Maximal Force}

A classic study by Doss \& Karpovich (1965) [4], using an isokinetic dynamometer, verified that ECC muscle actions performed with the elbow flexors, produced 40 and 14\% higher levels of force than CON and isometric actions, respectively. Sometime later, Rodgers \& Berger (1974) [5], using also isokinetic methods in the same muscle group, observed than the ECC torque was about $80 \%$ higher than CON torque. During the 90s, similar results were found by Hortobagyi \& Katch (1990) [6] , showing 22 and 60\% greater force values during ECC muscle actions if compared to CON contractions. Similarly, when free weights are used, ECC actions are still showing greater force values than CON. Although the reasons that may explain these discrepancies are still not well understood, higher maximal forces developed during ECC contractions seems to be related with muscle viscoelastic properties associated with motor unit activation. However, interpretation of these results should be done with caution since variables like exercise type and participants characteristics may influence the outcomes [7].

Colliander \& Tesch (1990) [8] described that the difference in maximal force values achieved between CON and ECC actions are much greater in women than in men. Thus, during ECC actions women could produce between 60 and $160 \%$ more force than during CON, while this range varied from 20 to $60 \%$ in men, depending on the exercise type performed. A recent study by Fernandez-Gonzalo et al.
(2011) [9] also suggests gender differences in muscle adaptations and maximal force manifestations induced by eccentric resistance training. However, gender differences during ECC resistance exercise are still not totally understood, although they may be related to several factors, such as muscle elastic component, central nervous system inhibition during maximal muscle contractions, motor unit activation and muscle quality [7].

\section{Lower Metabolic Cost}

Muscle groups activated and mode of muscle actions used are determined by exercise type. Generally, exercise type can be classified as either large or small muscle group exercises. Large muscle group exercises have a greater impact on the metabolic and hormonal responses to training $[10,11]$. Regardless of the size of muscle mass involved, ECC exercise shows lower metabolic cost than CON, despite of the higher forces produced during muscle lengthening [12]. Okamoto et al. (2006) [13] verified that the cardiovascular response induced by high-intensity contractions is smaller during ECC than CON muscle actions in a group of healthy young men. Similar results were obtained by Vallejo et al. (2006) [14] in elderly population. These data are consistent with investigations showing a relatively low adenosine adenosine triphosphate (ATP) turnover and reduced concentration of metabolites (e.g. ammonia and lactate) during ECC exercise $[15,16]$. The lower metabolic cost of ECC actions compared with CON may have different explanations. Thus, lower muscular activation [17], or lower phosphocreatine/creatine ( $\mathrm{PCr} / \mathrm{Cr}$ ) ratio [16] may help to explain the lower metabolic cost of ECC exercise. Another factor that could contribute to this response might be a greater contribution of elastic components in the muscle-tendon complex during ECC actions [16]. This lower metabolic cost is usually reflected in lower fatigability during ECC exercise.

*Corresponding author: Fernanda de Souza-Teixeira, Institute of Biomedicine University of León, Spain, Tel: +55-53-81185133; E-mail: fsout@unileon.es

Received December 12, 2011; Accepted May 25, 2012; Published May 29, 2012

Citation: de Souza-Teixeira F, de Paz JA (2012) Eccentric Resistance Training and Muscle Hypertrophy. J Sport Medic Doping Studie S1:004. doi:10.4172/2161-0673. S1-004

Copyright: (C) 2012 de Souza-Teixeira F, et al. This is an open-access article distributed under the terms of the Creative Commons Attribution License, which permits unrestricted use, distribution, and reproduction in any medium, provided the original author and source are credited. 


\section{Decreased fatigability and muscle activation}

Tesch et al. [18] found that electromiographic frequency signal decreased $40 \%$ after thirty-two isokinetic CON knee extensors contractions, while the signal did not decrease after thirty-two ECC actions. With a different protocol, Kay et al. [19] also found a decreased torque associated with a decreased electromyographic frequency signal after a hundred seconds of CON and isometric (ISO) contractions, whereas no change in this two variables was found after ECC actions. Furthermore, perception of fatigue is usually lower after ECC exercise than after combined CON/ECC exercise [20]

Studies using electromyography (EMG) analysis show that muscle activity during ECC muscle actions is lower than during ISO or CON exercise $[17,18,21,22]$, despite of the higher levels of force obtained during ECC actions. These data imply that voluntary maximal activation of muscle is not possible to be achieved during ECC actions. Some authors suggested that this reduction in muscle activation could be due to less activation of recruited fibers, although it also seems to be related with a characteristic fiber activation pattern [23]

Supporting data suggesting decreased fatigability during ECC exercise is provided by Roig et al. [24] The authors described that, when comparing peak values of strength during dynamic actions (CON vs. ECC), ECC strength is longer maintained than CON strength. However, the mechanisms explaining this response are not clear yet.

\section{Muscle Damage}

Muscle lengthening induces sarcomere stretching and, if the stress threshold is exceeded, sarcomeres may break or "pop" [23]. Furthermore, according to the hypothesis that ECC actions involve fewer motor units than $\mathrm{CON}$ actions to produce a given force, the active muscle fibers may experience greater stress (force per unit area), which would disrupt sarcolemmal, sarcoplasmic reticular, and myofibrillar structures $[25,26]$. This response is usually known as muscle damage.

Exercise induced muscle damage is a frequent response after unaccustomed or high intensity/duration exercise. Current symptoms of exercise induced muscle damage include stiffness, soreness and force reduction. Increase creatinkinasa, muscle troponin I, myoglobin and heavy myosin chain are some of the metabolic consequences of this kind of muscle damage [27].

Recently, Pitulainen et al. [28] described that the potential action propagation in affected fibers was reduced to a greater extent after ECC exercise if compared to CON. Furthermore, independently of the underlying mechanisms of ECC exercise induced muscle damage, it is accepted that this damage can prevail for long periods if frequency and intensity of exercise do not allow muscle regeneration and adaptation [29].

Response to myotrauma is usually followed by an acute inflammatory response. Once damage is detected, neutrophils migrate to the area of microtrauma. Damaged fibers release several agents that attract macrophages and lymphocytes to the injury site. Macrophages remove cellular debris trying to maintain the fiber's ultrastructure, while at the same time they produce cytokines that activate myoblasts, macrophages and lymphocytes. This response triggers the release of various growth factors that regulate satellite cell proliferation and differentiation [11]

\section{Eccentric Resistance Training and Hypertrophy}

Hypertrophy, defined as an increase in muscle size, is a general topic within several fields, like health, fitness and sports. It is widely accepted that hypertrophy is directly related to workload and tension developed [30]. Schoenfeld (2010) [11] indicated that hypertrophy is stimulated by three primary factors: mechanical tension, muscle damage and metabolic stress. However, the precise role of each of these three factors has not been fully elucidated [31].

As previously indicated, ECC exercise is a powerful triggering factor for damage to contractile and structural components of skeletal muscle [32]. This damage induces a cascade of physiological processes resulting in activation of satellite cells, which are located at the basal lamina that surrounds a myofibre. Satellite cells are not only activated to repair and maintain the muscle milieu, but also to increase the number of myonuclei. These two responses play a critical role in muscle regeneration and hypertrophy [32]. However, as cited by McCarthy et al. [33], the necessity of satellite cells for muscle hypertrophy is an important unresolved question.

In one study performed by LaStayo et al. [34] a significant increase of $6 \%$ in muscle mass was found without significant alterations on the inflammatory response. The study was designed to analyze the role of inflammation on muscle hypertrophy. They carried out an eccentric training program of 11 weeks with elderly subjects. Given the results they found, they concluded that neither damage, nor inflammation, appeared to be prerequisites to induce anabolic responses resulting in muscle growth in elderly individuals.

In a recent study by Flann et al. [35], similar results have been described in young adults. This study was designed to challenge the hypothesis that affirms that symptomatic damage is a necessary precursor for muscle remodeling. The authors compared two groups performing the same exercise with the same total work, but one of the groups had previously followed a familiarization process aimed to avoid any muscle damage during the "real" training. No differences between groups were found for muscle size or strength gains. Furthermore, the significant increases in mean cross-sectional area and strength were similar in both groups. Therefore, this study concluded that muscle hypertrophy can take place independently of any discernible sing of muscle damage.

Norrbrand et al. [36] using a gravity-independent resistance exercise system (instrument that offers resistance during coupled CON/ECC actions by using the inertia of a rotating flywheel), studied the changes in muscle activation and performance in response to 5 weeks of resistance training with or without eccentric overload. They described higher muscle activity during ECC actions with flywheel training (ECC overload) compared to standard weight lifting in healthy men. These results are supported by Tesch et al. [37] who also found a significant increase of muscle activity after five weeks of flywheel training in middle-aged men and women. The increase muscle activity was associated by the authors to an $11 \%$ increase in strength and $6 \%$ in muscle mass found in the same subjects. Taking into account data from these two studies [36,37], it seems that mechanical stress plays a critical role in muscle hypertrophy processes.

Mechanical loading is a critical stimulus to increase strength and size of skeletal muscle [3]. Thus, as a result of the ability to generate greater maximal forces during ECC actions, ECC training induces greater muscle hypertrophy than CON training [30]. Human studies show greater skeletal muscle protein synthesis following bouts of maximal ECC than CON exercise [38]. Furthermore, acute resistance exercise comprising only ECC or CON actions, elicited similar rate of protein synthesis despite the markedly less relative mechanical load employed in the ECC mode [39]. 
Hortobagyi et al. [40] studied the differences in muscle mass recovery after immobilization using three different training protocols: isolated CON, isolated ECC and coupled CON/ECC training. They found greater hypertrophy, in a shorter period, with ECC training, although they argued that their results could be related to a larger amount of work done during ECC muscle actions. However, it has been shown that muscle mass gains after ECC training are still greater than those after CON training even when the same absolute ECC and CON force levels are used [41]. Smith and Rutherford (1995) [42] suggested that metabolic cost and not high forces, may be the stimuli for muscle hypertrophy and strength gains following high-resistance training. However, this hypothesis still needs to be confirmed.

Another study [43] compared ECC and traditional rehabilitation training during the first fifteen weeks following anterior cruciate ligament reconstruction. They found greater short-term increases in muscle mass and strength in the ECC training group. Thus, muscle mass gains reported for quadriceps muscle were $10 \%$ greater for the ECC training group.

Walker et al. (1998) [44] studied the effects of CON and CON/ECC resistance training modes on muscle bioenergetics and cross-sectional area (CSA) of M.gastrocnemius using nuclear magnetic resonance in sixteen healthy young volunteers. They found significant increases in CSA and PCr/Pi and PCr/ATP resting ratios only in the CON/ECC group. They suggested that the improved oxidative mechanical power output could be due mainly to a greater muscle CSA.

However, a study with rats failed to find an association between the total amount of force generated during each contraction (ISO, $\mathrm{CON}$ and ECC) and hypertrophy response [45]. Since voluntary resistance training in humans is complicated by factors such as the potential for motor learning, the authors claimed that discrepancies between human and animal data may be explained by factors such as neural adaptation. Indeed, neural adaptations in ECC actions seem to be different compared with CON actions. Roig et al. [24] mentioned several characteristic of ECC muscle actions when compared with CON actions that included broader and faster cortical activity as movements are being executed, inversed motor unit activation pattern, increased cross-education effect, faster neural adaptations secondary to resistance training, attenuated muscle sympathetic nerve activity, reduced EMG amplitude at similar force levels, and greater EMG signal prior to the onset of movement.

A recent meta-analysis [24] analyzing the effects of ECC versus CON resistance training on muscle mass in healthy adults suggested that ECC exercise is more effective than CON exercise in increasing muscle mass. However, there are studies showing conflicting results regarding muscle mass gains after ECC versus CON. Nevertheless, it is necessary to take into account that results may differ depending on the methods used to assess muscle mass (muscle girth, dual $x$-ray absorptiometry (DEXA), ultrasound, magnetic resonance image (MRI) or computerized tomography (CT)). However, there are examples of conflicting results even when the same methodology to track muscle mass changes was used. Thus, using MRI we can find studies showing greater hypertrophy with ECC than CON $[46,47]$ as well as studies failing to show hypertrophy response after ECC training [48]. Since variables like studied population and type of intervention were comparable among those studies, the reasons for these discrepancies remain unclear.

Besides the mode of muscle action (ISO, CON or ECC), the magnitude of the training induced increase in CSA depends on several factors, including the initial strength of the individual, the durations of the training program, and the training technique used [49]. In novice subjects, for example, 6 weeks of ISO training increased the CSA of the elbow flexor muscles by about 5\% [50], whereas 8 weeks of ISO training increased the CSA of M.quadriceps femoris by 15\% [51]. Similarly, 60 days of isokinetic training at $2.1 \mathrm{rad} / \mathrm{s}$ with the knee extensors increased the CSA of M.quadriceps femoris by $9 \%$ [52]. Furthermore, 19 weeks of $\mathrm{ECC} / \mathrm{CON}$ knee extensor exercises produced a greater increase in CSA than exercises that involved only $\mathrm{CON}$ actions [53]. In contrast, twentyfour weeks of dynamic training by experienced body builders failed to elicit an increase in the CSA of muscle fibers in Biceps brachii [54] Given all the variables that can influence the hypertrophy response of a strength training program, and the controversial results found in ECC training studies, research comparing these variables in the hypertrophy response to ECC training seems mandatory.

\section{Conclusion}

Increased muscle cross-sectional area following resistance training occurs when the rate of protein synthesis is greater than protein degradation [32].

Considering the different qualities that eccentric muscle actions present compare to isometric or concentric muscle actions, it is theoretically possible that the benefits eccentric actions present may improve resistance training programs increasing several performance factors. The characteristics of eccentric actions include greater gains of muscle size and strength, decreased muscle soreness, and improvement of neural factors. Furthermore, eccentric exercise requires a lower metabolic cost than concentric or isometric exercise. Thus, the special characteristics of eccentric actions are becoming an important field of research trying to increase the positive outcomes of strength training while, at the same time, reduce the time of work [55].

On the other hand, negative aspects such damage and soreness, reduced neural reflexes, altered resting state and acute strength losses should be considered, and minimized, in an eccentric training programs.

Collectively results from studies cited in this short review suggest that eccentric muscle actions and high mechanical loads are essential stimuli to optimize exercise-induced muscle hypertrophy. In fact, several studies suggest that resistance training protocols comprising eccentric high-forces promote greater muscle hypertrophy than programs using concentric actions only. However, more studies involving eccentric resistance training are needed to fully elucidate its potential for exercise prescription.

\section{References}

1. Ryschon TW, Fowler MD, Wysong RE, Anthony AR, Balaban RS (1997) Efficiency of human skeletal muscle in vivo: comparison of isometric, concentric, and eccentric muscle action. J Appl Physiol 83: 867-874.

2. Stauber WT (1989) Eccentric action of muscles: physiology, injury and adaptation. Exerc Sport Sci Rev 17: 157-185.

3. Hortobayi T (2003) The positives of negatives: clinical implications of eccentric resistance exercise in old adults. J Gerontol A Biol Sci Med Sci 58: M417-M418.

4. Doss WS, Karpovich PV (1965) A comparison of concentric, eccentric, and isometric strength of elbow flexors. J Appl Physiol 20: 351-353.

5. Rodgers KL, Berger RA (1974) Motor-unit involvement and tension during maximum, voluntary concentric, eccentric, and isometric contractions of the elbow flexors. Med Sci Sports 6: 253-259.

6. Hortobagyi T, Katch FI (1990) Eccentric and concentric torque-velocity relationships during arm flexion and extension. Influence of strength level. Eur J Appl Physiol Occup Physiol 60: 395-401.

7. Hollander DB, Kraemer RR, Kilpatrick MW, Ramadan ZG, Reeves GV, et 
al. (2007) Maximal eccentric and concentric strength discrepancies between young men and women for dynamic resistance exercise. J Strength Cond Res 21: $34-40$.

8. Colliander EB, Tesch PA (1990) Effects of eccentric and concentric muscle actions in resistance training. Acta Physiol Scand 140: 31-39.

9. Fernandez-Gonzalo R, Bresciani G, de Souza-Teixeira F, Hernandez-Murua JA, Jimenez-Jimenez R, et al. (2011) Effects of a 4-week eccentric training program on the repeated bout effect in young active women. J Sports Sci Med 10: $692-699$

10. Spiering BA, Kraemer WJ, Anderson JM, Armstrong LE, Nindl BC, et al. (2008) Resistance exercise biology. Manipulation of resistance exercise programme variables determines the responses of cellular and molecular signalling pathways. Sports Med 38: 527-540.

11. Schoenfeld BJ (2010) The mechanisms of muscle hypertrophy and their application to resistance training. J Strength Cond Res 24: 2857-2872.

12. LaStayo PC, Woolf JM, Lewek MD, Snyder-Mackler L, Reich T, et al. (2003) Eccentric muscle contractions: their contribution to injury, prevention, rehabilitation, and sport. J Orthop Sports Phys Ther 33: 557-571.

13. Okamoto T, Masuhara M, Ikuta K (2006) Cardiovascular responses induced during high-intensity eccentric and concentric isokinetic muscle contraction in healthy young adults. Clin Physiol Funct Imaging 26: 39-44.

14. Vallejo AF, Schroeder ET, Zheng L, Jensky NE, Sattler FR (2006) Cardiopulmonary responses to eccentric and concentric resistance exercise in older adults. Age and Ageing 35: 291-297.

15. Horstmann T, Mayer F, Maschmann J, Niess A, Roecker K, et al. (2001) Metabolic reaction after concentric and eccentric endurance-exercise of the knee and ankle. Med Sci Sport Exerc 33: 791-795.

16. Roig M, Shadgan B, Darlene Reid W (2008) Eccentric exercise in patients with chronic health conditions: a systematic review. Physiother Can 60: 146-160.

17. Babault N, Pousson M, Ballay Y, Van Hoecke J (2001) Activation of human quadriceps femoris during isometric, concentric, and eccentric contractions. J Appl Physiol 91: 2628-2634.

18. Tesch PA, Dudley GA, Duvoisin MR, Hather BM, Harris RT (1990) Force and EMG signal patterns during repeated bouts of concentric or eccentric muscle actions. Acta Physiol Scand 138: 263-271.

19. Kay D, St Clair Gibson A, Mitchell MJ, Lambert MI, Noakes TD (2000) Different neuromuscular recruitment patterns during eccentric, concentric and isometric contractions. J Electromyogr Kinesiol 10: 425-431.

20. Miller PC, Hall EE, Chmelo EA, Morrison JM, DeWitt RE, et al. (2009) The influence of muscle action on heart rate, RPE, and affective responses after upper-body resistance exercise. J Strength Cond Res 23: 366-372.

21. Beltman JG, Van der Vliet MR, Sargeant AJ, de Haan A (2004) Metabolic cost of lengthening, isometric and shortening contractions in maximally stimulated rat skeletal muscle. Acta Physiol Scand 182: 179-187.

22. Gruber M, Linnamo V, Strojnik V, Rantalainen T, Avela J (2009) Excitability at the motoneuron pool and motor cortex is specifically modulated in lengthening compared to isometric contractions. J Neurophysiol 101: 2030-2040.

23. Enoka RM (1996) Eccentric contractions require unique activation strategies by the nervous system. J Appl Physiol 81: 2339-2346.

24. Roig M, O’Brien K, Kirk G, Murray R, McKinnon P, et al. (2009) The effects of eccentric versus concentric resistance training on muscle strength and mass in healthy adults: a systematic review with meta-analysis. Br J Sports Med 43: 556-568.

25. Warren GL, Hayes DA, Lowe DA, Armstrong RB (1993) Mechanical factors in the initiation of eccentric contraction-induced injury in rat soleus muscle. $J$ Physiol 464: 457-475.

26. Child RB, Saxton JM, Donnelly AE (1998) Comparison of eccentric knee extensor muscle actions at two muscle lengths on indices of damage and angle-specific force production in humans. J Sports Sci 16: 301-308.

27. Tee JC, Bosch AN, Lambert MI (2007) Metabolic consequences of exerciseinduced muscle damage. Sports Med 37: 827-836.

28. Piitulainen H, Holobar A, Avela J (2012) Changes in motor unit characteristics after eccentric elbow flexor exercise. Scand J Med Sci Sports 22: 418-429.

29. Krentz JR, Farthing JP (2010) Neural and morphological changes in response to a 20-day intense eccentric training protocol. Eur J Appl Physiol 110: 333-340.
30. Farthing JP Chilibeck PD (2003) The effects of eccentric and concentric training at different velocities on muscle hypertrophy. Eu J Appl Physiol 89: 578-586.

31. Ochi E, Nakazato K, Ishii N (2011) Muscular hypertrophy and changes in cytokine production after eccentric training in the rat skeletal muscle. J Strength Cond Res 25: 2283-2292.

32. Coffey VG, Hawley JA (2007) The molecular bases of training adaptation Sports Med 37: 737-763.

33. McCarthy JJ, Mula J, Miyazaki M, Erfani R, Garrison K, et al. (2011) Effective fiber hypertrophy in satellite cell-depleted skeletal muscle. Development 138 3657-3666.

34. LaStayo P, McDonagh P, Lipovic D, Napoles P, Bartholomew A, et al. (2007) Elderly patients and high force resistance exercise--a descriptive report: can an anabolic, muscle growth response occur without muscle damage or inflammation' J Geriatr Phys Ther 30: 128-134

35. Flan KL, LaStayo PC, McClain DA, Hazel M, Lindstedt SL (2011) Muscle damage and muscle remodeling: no pain, no gain? J Exp Biol 214: 674-679.

36. Norrbrand L, Pozzo M, Tesch PA (2010) Flywheel resistance training calls for greater eccentric muscle activation than weight training. Eur J Appl Physiol 110: 997-1005.

37. Tesch PA, Ekberg A, Lindquist DM, Trieschmann JT (2004) Muscle hypertrophy following 5-week resistance training using a non-gravity-dependent exercise system. Acta Physiol Scand 180: 89-98.

38. Moore DR, Phillips SM, Babraj JA, Smith K, Rennie MJ (2005) Myofibrillar and collagen protein synthesis in human skeletal muscle in young men after maximal shortening and lengthening contractions. Am J Physiol Endocrinol Metab 288: E1153-E1159.

39. Norrbrand L, Fluckey JD, Pozzo M, Tesch PA (2008) Resistance training using eccentric overload induces early adaptations in skeletal muscle size. Eur J App Physiol 102: 271-281.

40. Hortobagyi T, Dempsey L, Fraser D, Zheng D, Hamilton G, et al. (2000) Changes in muscle strength, muscle fibre size and myofibrillar gene expression after immobilization and retraining in humans. J Physiol 524: 293-304

41. Hortobagyi T, Hill JP, Houmard JA, Fraser DD, Lambert NJ, et al. (1996) Adaptative responses to muscle lengthening and shortening in humans. J App Physiol 80: 765-772.

42. Smith RC, Rutherford OM (1995) The role of metabolites in strength training I. A comparison of eccentric and concentric contractions. Eur J Appl Physio Occup Physiol 71: 332-336.

43. Gerber JP, Marcus RL, Dibble LE, Greis PE, Burks RT, et al. (2008) Effects of early progressive eccentric exercise on muscle size and function after anterior cruciate ligament reconstruction: a 1-year follow-up study of a randomized clinical trial. Phys Ther 89: 51-59.

44. Walker PM, Brunotte F, Rouhier-Marcer I, Cottin Y, Casillas JM, et al. (1998) Nuclear magnetic resonance evidence of different muscular adaptations after resistance training. Arch Phys Med Rehabil 79: 1391-1398.

45. Adams GR, Cheng DC, Haddad F, Baldwin KM (2004) Skeletal muscle hypertrophy in response to isometric, lengthening, and shortening training bouts of equivalent duration. J Appl Physiol 96: 1613-1618.

46. Higbie EJ, Cureton KJ, Warren GL, Prior BM (1996) Effects of concentric and eccentric training on muscle strength, cross-sectional area, and neural activation. J Appl Physiol 81: 2173-2181.

47. Seger JY, Thorstensson A (2005) Effects of eccentric versus concentric training on thigh muscle strength and EMG. Int J Sports Med 26: 45-52.

48. Blazevich AJ, Cannavan D, Coleman DR, Horne S (2007) Influence of concentric and eccentric resistance training on architectural adaptations in human quadriceps muscles. J Appl Physiol 103: 1565-1575.

49. Enoka RM (2002) Neuromechanics of human movement. Human kinetics $3^{\text {rd }}$ Edition.

50. Davies J, Parker DF, Rutherford OM, Jones DA (1988) Changes in strength and cross sectional area of elbow flexors as a result of isometric strength training. Eur J Appl Physiol 57: 667-670.

51. Garfinkel S, Cafarelli E (1992) Relative changes in maximal force, EMG, and muscle crosssectional area after isometric training. Med Sci Sports Exerc 24 1220-1227. 
Citation: de Souza-Teixeira F, de Paz JA (2012) Eccentric Resistance Training and Muscle Hypertrophy. J Sport Medic Doping Studie S1:004. doi:10.4172/2161-0673.S1-004

52. Narici MV, Roi GS, Landoni L, Minetti AE, Ceretelli P (1989) Changes in force, cross-sectional area and neural activation during strength training and detraining of the human quadriceps. Eur J Appl Physiol 59: 310-319.

53. Hather BM, Tesch PA, Buchanan P, Dudley GA (1991) Influence of eccentric actions on skeletal muscle adaptations to resistance training. Acta Physio Scand 143: 177-185.
54. Always SE, Grumbt WH, Stray-Gundersen J, Gonyea WJ (1992) Effects of resistance training on elbow flexors of highly competitive bodybuilders. J Appl Physiol 72: 1512-1521.

55. Hortobagyi T, Barrier J, Beard D, Braspennincx J, Koens P, et al. (1996) Greater initial adaptations to submaximal muscle lengthening than maxima shortening. J Appl Physiol 81: 1677-1682.

This article was originally published in a special issue, Muscle Hypertrophy handled by Editor(s). Dr. Jennifer A. Bunn, Campbell University, USA 